\title{
Computational Fluid Dynamics Modeling in Aortic Diseases
}

pISSN 2508-707X / elSSN 2508-7088 https://doi.org/10.22468/cvia.2018.00073 CVIA 2018;2(2):58-64

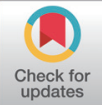

Received: March 20, 2018

Revised: April 9, 2018

Accepted: April 10, 2018

Corresponding author

Takuya Ueda, MD, PhD

Department of Diagnostic Radiology,

Tohoku University Hospital,

1-1 Seiryo, Aoba, Sendai 980-8574, Japan

Tel: 81-22-717-7312

Fax: 81-22-717-7316

E-mail: takuedarad@tohoku.ac.jp

\author{
Takuya Ueda', Hiroshi Suito ${ }^{2}$, Hideki Ota', Kei Takase' \\ 'Department of Diagnostic Radiology, Tohoku University Hospital, Sendai, Japan \\ ${ }^{2}$ Advanced Institute for Materials Research, Tohoku University, Sendai, Japan
}

\begin{abstract}
In recent years, computational fluid dynamics (CFD) has attracted considerable attention in cardiovascular medicine. In addition to traditional assessment based on anatomical information, the CFD-based approach provides an opportunity to gain novel insights into vascular pathophysiology by exploring the relationship between structure and biomechanical forces in flow dynamics. As recently CFD modeling is widely used in clinical situations, it is very important for clinicians to understand the basic principles, benefits, limitations, and pitfalls of CFD modeling. In this paper, we present an overview of CFD modeling and its application to aortic diseases, and primarily to aortic aneurysms.
\end{abstract}

Key words Cardiovascular disease - Aorta - Aneurysm - Computational fluid dynamics.

\section{INTRODUCTION}

Originally, computational fluid dynamics (CFD) was a mechanical engineering method for comprehensive analysis of fluid flow, heat transfer, and associated phenomena with the use of computer-based simulations. Recently, it has become a widely adopted methodology for solving complex problems in many modern engineering fields. The CFD-based approach is used for understanding the relationship between structure and flow by solving systems of partial differential equations to simulate fluid flow. Advances in medical imaging and computational methods provide an opportunity to apply the CFD-based approach in clinical medicine. Cardiovascular imaging is one of the most common medical applications of CFD modeling. Although CFD modeling is essentially studied by applied mathematicians and engineers who are specialists in fluid mechanics, as CFD modeling continues to spread into clinical medicine it is very important for clinicians to understand the basic principles, benefits, limitations, and pitfalls of CFD modeling. In this paper, we present an overview of CFD modeling and its application to aortic diseases, and primarily to aortic aneurysms.

\section{FRAMEWORK OF CFD MODELING}

CFD modeling is performed by solving differential equations to simulate fluid flow. The construction and solution of a

(a) This is an Open Access article distributed under the terms of the Creative Commons Attribution Non-Commercial License (http://creativecommons.org/licenses/bync/4.0) which permits unrestricted non-commercial use, distribution, and reproduction in any medium, provided the original work is properly cited.
CFD model involves several steps.

\section{Geometrical discretization for CFD modeling}

The first step of CFD modeling is to define the geometry of the region of interest to simulate the fluid flow. Geometrical extraction converts medical images into numerical geometries that define the physical boundaries of the model region of interest. A variety of medical imaging techniques can be used for geometrical extraction, including ultrasound, CT, MRI, and Xray angiography. Spatial discretization, or "meshing," divides the geometry into a number of discrete volumetric elements or cells. Temporal discretization divides the system into discrete time steps [1]. As the accuracy of a solution of a CFD model is essentially dependent on geometry extraction-namely, the spatial resolution and image quality of medical imaging-medical imaging must provide sufficient anatomical resolution to enable subsequent segmentation and data extraction. Most of the time spent on CFD modeling is devoted to this geometrical extraction process [2]. In cardiovascular systems, computational imaging tools may confer grid generation information, but there are limitations in that the resolution of current imaging tools is still low and the geometry varies according to the cardiac cycle [2]. Additionally, the time required for the computational solution of a CFD system depends on the resolution of the grid. Recently, high-performance computing systems have addressed many of the challenges facing huge, high-resolution CFD systems incorporating detailed geometrical data, thereby relieving CFD modeling of the long times required to solve complicated systems. 


\section{Definition of fluid properties}

Prior to performing a CFD simulation of a cardiovascular system, the essential fluid properties of blood need to be clearly defined. Most non-biological fluids approximate Newtonian fluids, which result in a constant viscosity. The various components of blood, including plasma, blood cells, and other carried material, tend to cause blood to behave in a non-Newtonian manner in various degree depends on the biological condition and anatomical scale of vessels, where the blood's viscosity changes with the shear rate of the flow [1]. Thus, the correct viscosity model, consisting of a mathematical equation, should be selected based on the range of shear rates in the system. For example, when the shear rate is sufficiently high, such as in an aorta or in large vessels, blood flow exhibits Newtonian flow behavior, but the nonNewtonian behavior of the fluid cannot be ignored in small vessels [1]. The rate of change in velocity from along the vessel wall to the center of the vessel is proportional to the wall shear stress (WSS) [1].

\section{Boundary conditions}

Because it is impossible to discretize the entire cardiovascular system in clinical situations, the region to be analyzed will have at least one inlet and one outlet of flow. To enable CFD modeling, boundary conditions of the flow, namely the physiological conditions at the inlet and outlet boundaries, must be specified [1]. Boundary conditions are a set of applied physiological parameters that may vary over time, such as such as blood pressure, blood flow velocity, and temperature. Boundary conditions may be based on patient-specific data, population data, physical models, or assumptions [1,2].

\section{Numerical solution}

After the pre-processing steps discussed above, the next step in CFD modeling is finding a numerical solution. Almost all CFD analyses solve the Navier-Stokes equations and continuity equations that govern fluid motion. A variety of numerical solution techniques are available, such as finite difference, finite element, finite volume, and spectral methods [2]. In a typical three dimensional (3D) cardiovascular simulation, millions of non-linear partial differential equations are solved simultaneously, and repeatedly, over all elements, at all time-steps. Once the numerical solution is found, the CFD model describes the condition of the flow, such as the pressure and velocity fields, over all elements and at each time-step.

\section{Post-processing}

The generated results of a numerical solution of a CFD model by a computer cannot be assessed directly. Also, some specific CFD parameters that are associated with the histopathological condition of the disease are of interest in the medical simulation.
Therefore, some post-processing is required to extract and visualize relevant data. After this process, the researcher can easily understand the simulation results. Many visualization tools have been developed, including domain geometry and grid display, vector plots, line and shaded contour plots, two-dimensional and 3D surface plots, particle tracking, and color postscript outputs [2]. Various parameters of CFD modeling that are relevant to cardiovascular medicine are discussed in a later section.

\section{CLINICAL SIGNIFICANCE OF CFD MODELING FOR AORTIC ANEURYSMS}

For many years, the size of the aorta has been the principle decision making criteria for intervention of an aortic aneurysm. Many guidelines for the treatment of aortic aneurysms advocate a maximal aortic diameter and clinical risk factors as the determination of the timing for intervention [3]. Many studies have suggested that some small aortic aneurysms may rupture prior to reaching $55 \mathrm{~mm}$ of maximum short diameter, which is the suggested threshold size for surgical intervention [4], whereas many aneurysms larger than the threshold never rupture. Therefore, appropriate predictors for the risk of rupture and the progression of an aneurysm, other than aortic size, would improve current treatment strategies by providing appropriative timing for intervention, while also reducing the expense and risk of unnecessary intervention.

One goal of CFD modeling in cardiovascular medicine is to understand the relationships between anatomical geometry (i.e., vascular anatomy), local hemodynamics (blood flow), fluid-structure interaction (FSI) including stress and pressure on the vessel wall, and the vascular pathology (development and progression of atherosclerosis, aneurysm, dissection, etc.) (Fig. 1). Several studies have suggested that hemodynamic forces may provide one of the most significant biomechanical effects underlying aneurysm development [5]. As measurement of in vivo hemodynamics assessment can be difficult and invasive, researchers are gradually focusing on the assessment of hemodynamics, using non-invasive CFD modeling to predict the risk of future rupture for aneurysms.

\section{HEMODYNAMIC FORCES RELEVANT TO AORTIC PATHOLOGY}

Clinical and experimental observations have indicated that various biomechanical conditions interactively influence the progression of an aortic aneurysm [6,7]. Fig. 2 illustrates the various hemodynamic forces that are relevant to biomechanical conditions in aortic pathologies. 


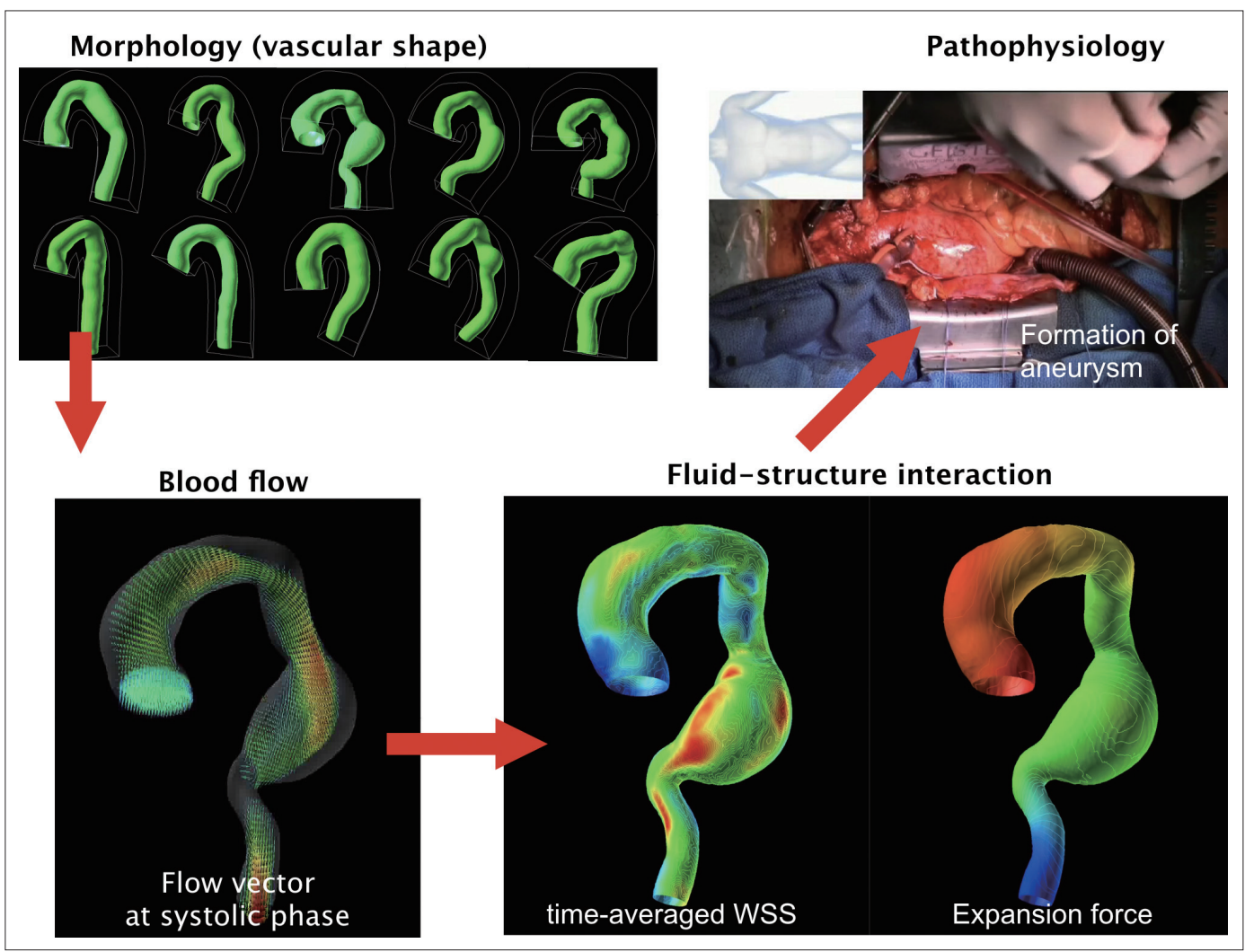

Fig. 1. CFD modeling: interactions between morphology, flow, and various biomechanical forces. CFD modeling depicts the interaction between vascular morphology and FSI to elucidate the pathophysiology of aortic disease. Differences in morphology (shape of the aorta) generate different blood flow patterns (flow dynamics). Due to the FSI, the vessel lumen is exposed to laminar WSS during the cardiac cycle. Also, pulsatile flow applies pressure to the wall and the vessels are distended during the cardiac cycle. Such continuous biomechanical forces can provoke pathophysiological conditions of aortic disease, such as an aneurysm. The aorta may rupture when the maximum distention force on the vessel wall exceeds the tensile strength of the wall. CFD: computational fluid dynamics, FSI: fluid-structure interaction, WSS: wall shear stress.

\section{Wall shear stress}

WSS, which is the tangential force of blood flowing on the endothelial surface of blood vessels, is the most significant of the hemodynamic forces in a blood vessel. The interactions of pulsatile blood flow with arterial structures generate complex hemodynamic forces on the vessel wall that exhibit spatial and temporal variation (Fig. 3) [8]. Endothelial cellular responses to these physical stimuli influence vessel wall homeostasis. Constant laminar blood flow with tangential WSS protects the vascular endothelium [9]. After fluctuations of WSS promote changes in biochemical signals, the biophysiological characters of vessel wall starts to degenerate. The degeneration of vessel wall will directly lead to the initiation and progression of some cardiovascular diseases, including aortic aneurysm [10] and coronary artery disease [11].

\section{Pitfalls in the assessment of various WSS parameters}

Although WSS is one of the most frequently studied hemodynamic parameters in cardiovascular diseases, careful attention should be paid to the interpretation of CFD modeling that is used in articles or in studies. As the pulsatile nature of blood flow produces complicated temporal and spatial WSS patterns during the cardiac cycle, various WSS parameters have been linked with cardiovascular complications, such as WSS at peak systole, time-averaged WSS, and time-averaged spatial WSS gradient. In addition, most studies rely on measures of WSS magnitude while little attention has been paid to the vectoral behavior of WSS [12]. One study suggests that the transverse WSS perpendicular to the flow direction is a more significant WSS parameter rather than the WSS magnitude [13].

A typical pitfall in WSS assessment is the fact that "the timeaverage of magnitude of WSS" and "the magnitude of time-averaged WSS" are different because WSS is a vector-valued quantity. The difference in values of "the time-average of magnitude of WSS" and "the magnitude of time-averaged WSS" becomes significant in flows with fluctuation where the directions of WSS vector changes with time. This pitfall is also discussed in the oscillatory shear index (OSI) section below.

\section{Oscillatory shear index}

OSI is another significant hemodynamic parameter used to assess WSS in that it has the time-averaged magnitude of WSS in 
the denominator. OSI quantifies the fluctuations of WSS from the primary flow direction during the cardiac cycle (Fig. 4). For example, blood flow with fluctuations possesses a high OSI even though its time-averaged WSS is low. Fluctuations of WSS disturb the vessel wall homeostasis and may induce aortic pathologies [10].

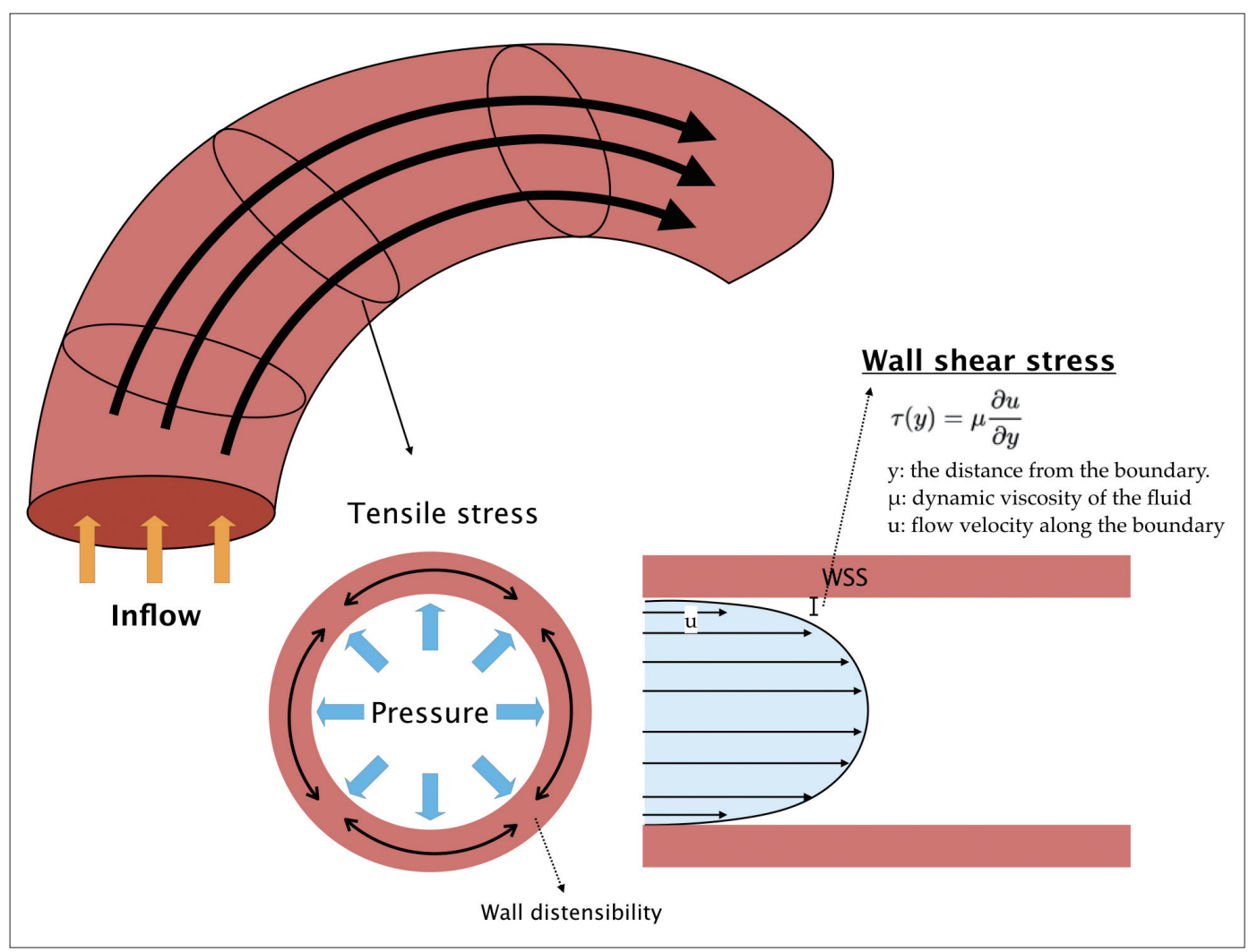

Fig. 2. Various hemodynamic forces of blood flow in the aorta. Schematic illustration of various hemodynamic forces of blood flow in the aorta. Through FSI, pulsatile aortic flow generates WSS (the tangential force exerted by moving blood along the vessel wall) and tensile stress (the perpendicular forces acting on the vascular wall) on the aortic wall during the cardiac cycle. FSI: fluid-structure interaction, WSS: wall shear stress.
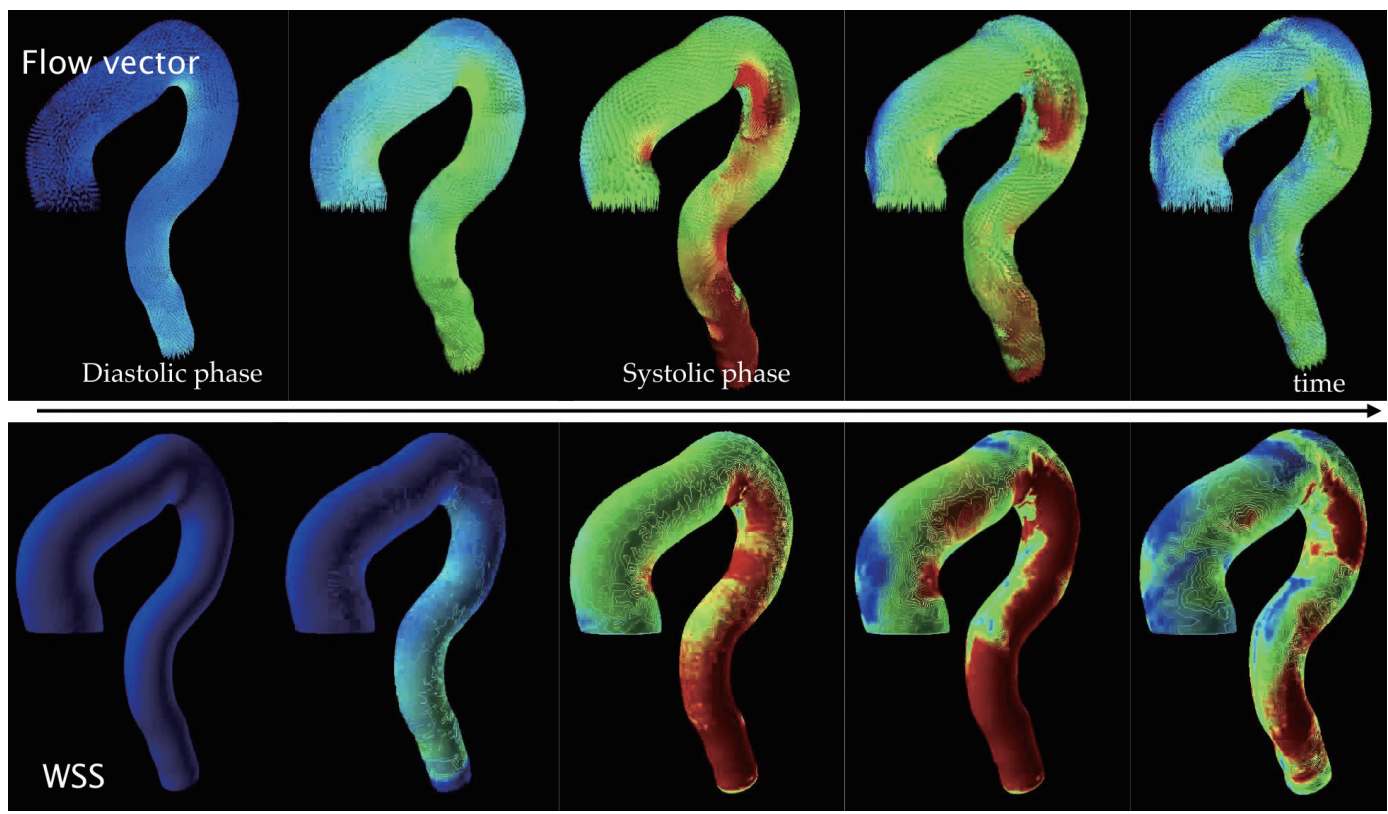

Fig. 3. Time course change of flow vector and WSS. The time course change of the flow vector of blood flow (upper row) and WSS (lower row). Note that the pulsatile nature of blood flow produces complicated temporal and spatial WSS patterns. WSS: wall shear stress. 


\section{Vessel wall strain and distensibility}

Recently, several researchers investigated other biomechanical forces aside from WSS for predicting the risk of rupture. Di Martino et al. [14] reported that maximum tissue stiffness was inversely correlated with wall strength, suggesting lower stiffness as a possible predictor of aneurysm rupture. Also, Wilson et al. suggested that distensibility at baseline and the change of the aorta during follow-up were correlated with the risk of rupture, in addition to the diastolic pressure and larger parameter [15]. Several ultrasound studies reported assessment of local wall strain of the whole abdominal aortic aneurysm in vivo [16]. Satriano et al. [17] proposed a 3D image-based approach to compute aortic wall strain maps in vivo, which was used for various imaging modalities. In terms of CFD, Stevens et al. [18] used a CFD method to assess how a change of flow-related biomechanical properties affects the wall stress and the related wall strain. Although further clinical studies are required, such hypotheses regarding vessel wall strain and wall distensibility would add more insight in predicting the precise risk for aneurysm rupture.

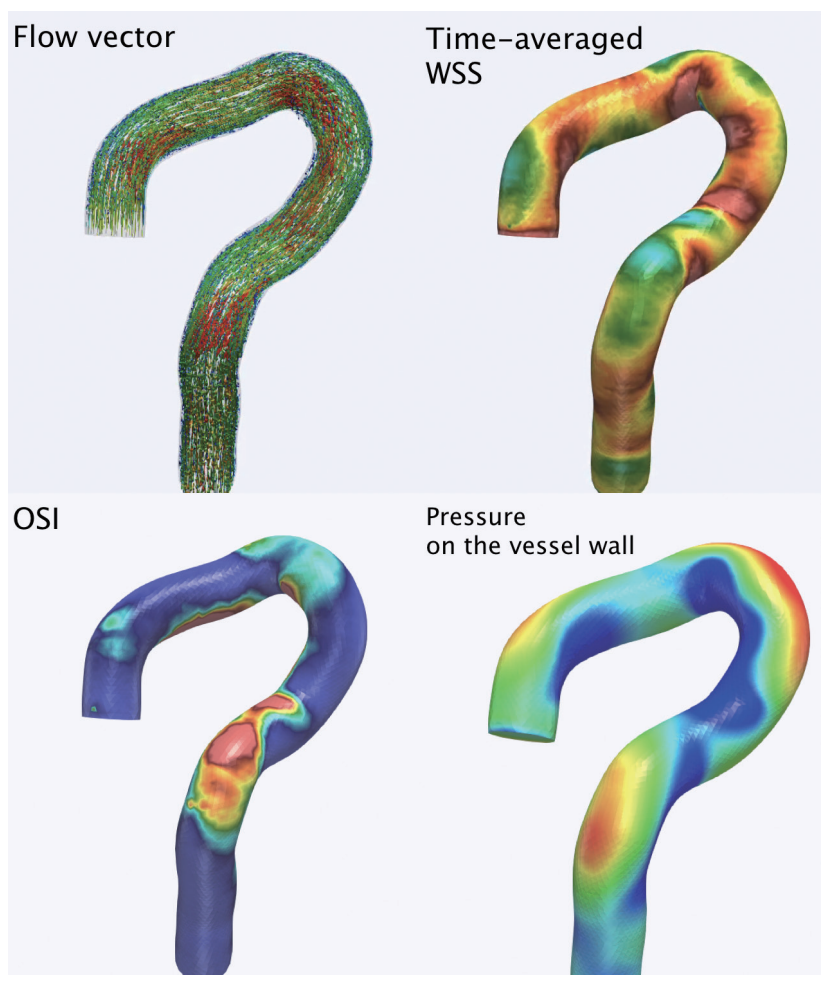

Fig. 4. Interrelationship between flow, time-averaged WSS, OSI, and pressure on the vessel wall (distention force). The interrelationship between flow vector at peak systolic phase, time-averaged WSS, OSI, and pressure on the vessel wall (distention force). The interactions of pulsatile blood flow with arterial structures generate complex hemodynamic forces on the vessel wall due to the fluidstructure interaction. Note that WSS is high and OSI is low in the distal aortic arch because of constant, laminar blood flow, while WSS is low and OSI is high in the thoraco-abdominal junction because of the fluctuation of the flow direction and the WSS vector during the cardiac cycle. WSS: wall shear stress, OSI: oscillatory shear index.
Fig. 4 illustrates the interrelationship between flow, WSS, OSI, and pressure on the vessel wall (i.e., distention force).

\section{REGIONAL HEMODYNAMIC DIFFERENCES RELEVANT TO AORTIC ANEURYSMS}

Few studies have investigated regional pathogenic risks of aortic pathologies. Even in different regions in the aorta that have the same diameter, the behavior of aortic dilatation differs depending on the location in the aorta. Usually, the abdominal aorta has a marked predilection for aneurysmal dilatation when compared to the thoracic aorta, where the infrarenal aorta is the most common site of aortic aneurysm formation. Different hemodynamic influences present along the length of the aorta may work in concert with other regional factors to explain this preferential distribution [6].

Region-specific structural differences are well recognized along the aorta. For example, the elastin-collagen ratio declines along the length of the aorta, reducing elasticity and wall motion [19]. Reduced distal aortic elasticity, in combination with augmented pressure due to pulse wave reflections from aortic bifurcation and other downstream arteries, may increase wall strain and aneurysm susceptibility [20].

Most relevant to aortic disease pathophysiology, and its predilection for the distal aortic segment, is the marked difference between aortic WSS in the proximal and distal aorta. In proximal aortic segments, flow is antegrade throughout the cardiac cycle, providing continuous antegrade laminar WSS [6]. As discussed above, constant laminar blood flow with normal WSS regulates homeostasis of the vascular endothelium [9]. In general, the time-averaged WSS is high, while the OSI is low in the distal thoracic aortic arch because of uniform laminar flow. In contrast, many experimental and numerical studies have indicated that multiple secondary reverse flows with vortex formation were observed in the late systolic and diastolic phases during the cardiac cycle in the distal abdominal aorta, while they were not observed in the thoracic and proximal aorta $[6,11,21]$. As a result, the time-averaged WSS is low and the OSI is high in the distal abdominal aorta when compared with proximal aorta. These distinct regional differences in hemodynamic influences may account for some component of the differential aneurysm risk noted between the thoracic and abdominal aortic segments.

Furthermore, there is individual variation in the location of aneurysmal formation among patients. Although the infrarenal aorta is the most common site of aortic aneurysm formation, some patients have an aneurysm in the distal aortic arch or in the thoraco-abdominal junction. Differences of anatomical geometry (shape of the aorta) can generate patient-specific flow patterns and FSIs during the cardiac cycle [22]. CFD modeling improves our understanding of variation in the location of an- 

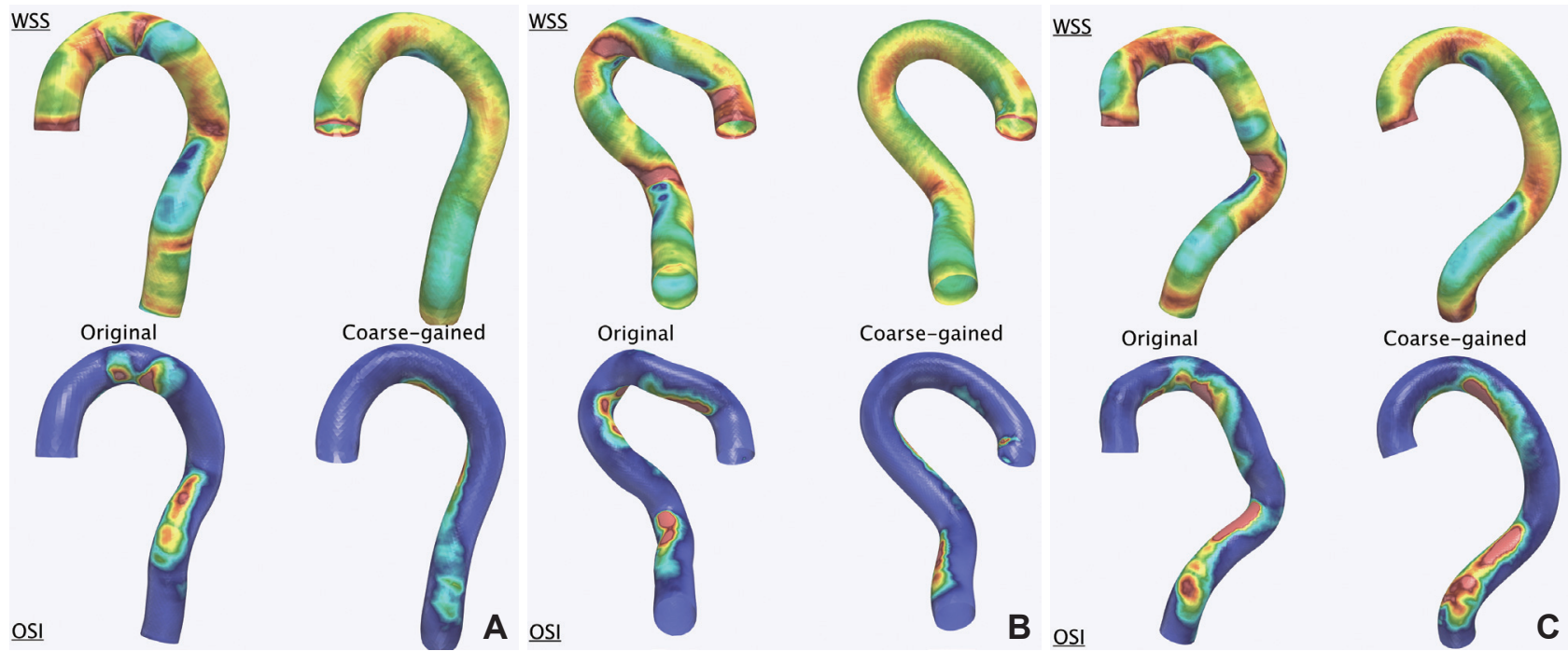

Fig. 5. Different patterns of anatomical geometry, flow, and various biomechanical forces. CFD modeling simulates time-averaged WSS and OSI in patients with an aneurysm on the (A) aortic arch, $(B)$ descending aorta or (C) abdominal aorta. To simulate the shape of the aorta before showing elongation and dilatation (a virtual pre-pathological condition), we generate an aortic shape with a uniform diameter and a coarse-grained curve by decreasing the number of control points in the Non-Uniform Rational B-Spline (NURBS) representation [24]. The original shape is shown on the left and the coarse-grained shapes are shown on the right. (A) CFD simulation of a patient with an aneurysm on the aortic arch showed a high OSI at the lesser curvature of the aortic arch both in the original shape and in the coarse-grained shape. (B) CFD simulation of a patient with an aneurysm on the descending aorta showed a high OSI at the descending aorta and at the thoracoabdominal junction in the coarse-grained shape. (C) CFD simulation of a patient with an abdominal aneurysm showed a high OSI at the thoraco-abdominal junction and at the abdominal aorta in the coarse-grained shape. CFD: computational fluid dynamics, OSI: oscillatory shear index.

eurysmal formation among individual patients (Fig. 5).

\section{LIMITATIONS AND PITFALLS OF CFD MODELING}

In spite of the many advantages of the clinical application of CFD modeling, and the variety of useful visualizations provided by CFD modeling, simulated CFD data should be thoroughly validated. The accuracy of the model is determined by the model's design and the quality of the input data. The validity of CFD modeling results depends significantly on the selection of appropriate geometry discretization, boundary conditions, fluid properties, and the numerical solution methodology [23]. Due to the many simplifications and assumptions taken into account in CFD modeling, the degree of accuracy of the results needs to be assessed prior to application in clinical practice [23].

In this sense, doctors should learn how to assess a CFD model and understand its inherent limitations and pitfalls. Particularly, doctors should make critical judgments about the computed results from the perspective of medical validity [2]. Some kind of clinical variation should be attempted in clinical trials.

\section{CONCLUSIONS}

The advancement of CFD modeling has presented a unique opportunity to provide new insights into vascular hemodynamics in the assessment of cardiovascular diseases. Patient- specific CFD modeling has the potential to provide a comprehensive understanding of the interaction between vascular morphology, blood flow, and FSI. Information about biomechanical forces in aortic pathologies may help to predict the risk of aortic aneurysm and to select appropriate treatment.

\section{Conflicts of Interest}

The authors declare that they have no conflict of interest.

\section{Acknowledgments}

This work was supported by JST CREST, Japan (grant number JPMJCR15D1).

\section{REFERENCES}

1. Wong KK, Wang D, Ko JK, Mazumdar J, Le TT, Ghista D. Computational medical imaging and hemodynamics framework for functional analysis and assessment of cardiovascular structures. Biomed Eng Online 2017;16:35.

2. Lee BK. Computational fluid dynamics in cardiovascular disease. Korean Circ J 2011;41:423-430.

3. Moll FL, Powell JT, Fraedrich G, Verzini F, Haulon S, Waltham M, et al.; European Society for Vascular Surgery. Management of abdominal aortic aneurysms clinical practice guidelines of the European society for vascular surgery. Eur J Vasc Endovasc Surg 2011;41 Suppl 1:S1-S58.

4. Elefteriades JA, Farkas EA. Thoracic aortic aneurysm clinically pertinent controversies and uncertainties. J Am Coll Cardiol 2010;55:841-857.

5. Hsiai TK. Mechanosignal transduction coupling between endothelial and smooth muscle cells: role of hemodynamic forces. Am J Physiol Cell Physiol 2008;294:C659-C661.

6. Dua MM, Dalman RL. Hemodynamic influences on abdominal aortic aneurysm disease: application of biomechanics to aneurysm pathophysiology. Vascul Pharmacol 2010;53:11-21.

7. Wang Y, Joannic D, Delassus P, Lalande A, Juillion P, Fontaine JF. Com- 
parison of the strain field of abdominal aortic aneurysm measured by magnetic resonance imaging and stereovision: a feasibility study for prediction of the risk of rupture of aortic abdominal aneurysm. J Biomech 2015;48:1158-1164.

8. Kwak BR, Bäck M, Bochaton-Piallat ML, Caligiuri G, Daemen MJ, Davies $\mathrm{PF}$, et al. Biomechanical factors in atherosclerosis: mechanisms and clinical implications. Eur Heart J 2014;35:3013-3020.

9. Wasserman SM, Topper JN. Adaptation of the endothelium to fluid flow: in vitro analyses of gene expression and in vivo implications. Vasc Med 2004;9:35-45.

10. Biasetti J, Hussain F, Gasser TC. Blood flow and coherent vortices in the normal and aneurysmatic aortas: a fluid dynamical approach to intra-luminal thrombus formation. J R Soc Interface 2011;8:1449-1461.

11. Chatzizisis YS, Coskun AU, Jonas M, Edelman ER, Feldman CL, Stone $\mathrm{PH}$. Role of endothelial shear stress in the natural history of coronary atherosclerosis and vascular remodeling: molecular, cellular, and vascular behavior. J Am Coll Cardiol 2007;49:2379-2393.

12. Arzani A, Shadden SC. Characterizations and correlations of wall shear stress in aneurysmal flow. J Biomech Eng 2016;138:014503.

13. Peiffer V, Sherwin SJ, Weinberg PD. Computation in the rabbit aorta of a new metric - the transverse wall shear stress - to quantify the multidirectional character of disturbed blood flow. J Biomech 2013;46:2651-2658.

14. Di Martino ES, Bohra A, Vande Geest JP, Gupta N, Makaroun MS, Vorp DA. Biomechanical properties of ruptured versus electively repaired abdominal aortic aneurysm wall tissue. J Vasc Surg 2006;43:570-576; discussion 576.

15. Wilson K, Bradbury A, Whyman M, Hoskins P, Lee A, Fowkes G, et al.
Relationship between abdominal aortic aneurysm wall compliance and clinical outcome: a preliminary analysis. Eur J Vasc Endovasc Surg 1998; 15:472-477.

16. Bihari P, Shelke A, Nwe TH, Mularczyk M, Nelson K, Schmandra T, et al. Strain measurement of abdominal aortic aneurysm with real-time 3D ultrasound speckle tracking. Eur J Vasc Endovasc Surg 2013;45:315-323.

17. Satriano A, Rivolo S, Martufi G, Finol EA, Di Martino ES. In vivo strain assessment of the abdominal aortic aneurysm. J Biomech 2015;48:354360 .

18. Stevens RRF, Grytsan A, Biasetti J, Roy J, Lindquist Liljeqvist M, Gasser TC. Biomechanical changes during abdominal aortic aneurysm growth. PLoS One 2017;12:e0187421.

19. Ailawadi G, Eliason JL, Upchurch GR Jr. Current concepts in the pathogenesis of abdominal aortic aneurysm. J Vasc Surg 2003;38:584-588.

20. Humphrey JD, Taylor CA. Intracranial and abdominal aortic aneurysms: similarities, differences, and need for a new class of computational models. Annu Rev Biomed Eng 2008;10:221-246.

21. Guzmán AM, Amon CH. Dynamical flow characterization of transitional and chaotic regimes in converging-diverging channels. J Fluid Mech 1996;321:25-57.

22. Suito H, Takizawa K, Huynh VQH, Sze D, Ueda T. FSI analysis of the blood flow and geometrical characteristics in the thoracic aorta. Comput Mech 2014;54:1035-1045.

23. Morris PD, Narracott A, von Tengg-Kobligk H, Silva Soto DA, Hsiao S, Lungu A, et al. Computational fluid dynamics modelling in cardiovascular medicine. Heart 2016;102:18-28.

24. Piegl L, Tiller W. The NURBS book. 2nd ed. Berlin: Springer, 1997. 